$16^{\text {th }}$ International Congress of Metrology, 06004 (2013)

DOI: $10.1051 /$ metrology/201306004

(c) Owned by the authors, published by EDP Sciences, 2013

\title{
Least squares best-fit geometric elements taking into account uncertainty structure
}

\author{
Ian M Smith ${ }^{1, a}$ and Alistair B Forbes ${ }^{1}$ \\ ${ }^{1}$ National Physical Laboratory, Hampton Road, Teddington, Middlesex, TW11 0LW, UK
}

\begin{abstract}
In coordinate metrology, a key activity is fitting a geometric surface to coordinate data. By far the most common fitting criterion has been ordinary least squares (OLS). OLS is appropriate if the covariance matrix associated with the data is a diagonal matrix. However, in recent years much effort has been devoted to developing more realistic uncertainty models for coordinate data and it is now timely to develop fitting algorithms that take into account this richer uncertainty structure. For many measuring systems, the uncertainty characterisation is such that the covariance matrix associated with coordinate data can be represented in a compact, factored form that makes explicit the contribution of random and systematic effects associated with the measuring system. This compact representation enables the least squares fitting algorithm to be implemented using essentially the same computational components as for the OLS fitting scheme.
\end{abstract}

\section{Introduction}

In coordinate metrology, a key activity is fitting a geometric surface to coordinate data. By far the most common fitting criterion has been ordinary least squares (OLS). OLS is appropriate if the covariance matrix associated with the data is a diagonal matrix. However, in recent years much effort has been devoted to developing more realistic uncertainty models for coordinate data and it is now timely to develop fitting algorithms that take into account this richer uncertainty structure. In fact, the effort in developing realistic uncertainty characterisations is only beneficial if the algorithms acting on the coordinate data use this extra information. For many measuring systems, the uncertainty characterisation is such that the covariance matrix associated with coordinate data can be represented in a compact, factored form that makes explicit the contribution of random and systematic effects associated with the measuring system. This compact representation enables the least squares fitting algorithm to be implemented using essentially the same computational components as for the OLS fitting scheme. In addition, the new algorithm can often give insight into the behaviour of the measuring system that gave rise to the measurement data. In particular, the algorithm can be used explicitly to determine uncertainties in coordinate metrology when the coordinate measuring system is used in a comparator mode.

In section 2 we discuss the uncertainty characterisation of measuring systems, while in sections 3 and 4 we discuss, respectively, ordinary least squares (OLS) and generalised least squares (GLS) surface fitting algorithms. Our summary and concluding remarks are given in section 5 .

\subsection{Notation}

Throughout this paper, we use the following notation to represent the coordinates associated with a set of data points in three dimensions. Given point coordinates $\boldsymbol{x}_{i}=\left(x_{i}, y_{i}, z_{i}\right)^{\mathrm{T}}, i \in I=\{1, \ldots, m\}, \boldsymbol{x}_{I}$ is the $3 m \times 1$ vector

$$
\boldsymbol{x}_{I}=\left(x_{1}, y_{1}, z_{1}, x_{2}, y_{2}, z_{2}, \ldots, x_{m}, y_{m}, z_{m}\right)^{\mathrm{T}} .
$$

\section{CMM kinematic model}

The kinematic error of a coordinate measuring machine (CMM) describes the systematic error behaviour of the CMM arising from non-ideal geometry relating to scale errors, straightness and rotational errors. The error behaviour is defined in terms of the 18 univariate functions $d_{x x}, d_{x y}, \ldots, e_{z z}$, six a function of $x, \operatorname{six}$ a function of $y$ and six a function of $z$. These error functions can be combined into a single error function $\boldsymbol{e}(\boldsymbol{x}, \boldsymbol{b})$ depending on parameters $\boldsymbol{b}=\left(b_{1}, \ldots, b_{p}\right)^{\mathrm{T}}$. The actual centre of the CMM probe, $\boldsymbol{x}^{*}$, is related to the measured probe centre $\boldsymbol{x}$ output by the CMM through a model equation of the form

$$
\boldsymbol{x}=\boldsymbol{x}^{*}+\boldsymbol{e}\left(\boldsymbol{x}^{*}, \boldsymbol{b}\right)+\boldsymbol{\epsilon},
$$

where $\boldsymbol{\epsilon}$ is a random effect. We make the assumption that $\boldsymbol{e}$ is sufficiently smooth so that $\boldsymbol{e}\left(\boldsymbol{x}^{*}, \boldsymbol{b}\right)$ can be approximated effectively by $\boldsymbol{e}(\boldsymbol{x}, \boldsymbol{b})$, i.e., the error function $\boldsymbol{e}$ evaluated at the measured coordinates $\boldsymbol{x}$.

Estimates of the kinematic error parameters $\boldsymbol{b}$ can be determined experimentally from the measurements of hole or ball plates $[9,11]$, for example. For a ball plate experiment, let $\boldsymbol{y}_{j}$ be the location of the $j$ th ball centre in a fixed frame of reference and let

$$
\boldsymbol{y}_{j, k}=T\left(\boldsymbol{y}_{j}, \boldsymbol{t}_{k}\right)=\boldsymbol{x}_{k, 0}+R^{\mathrm{T}}\left(\boldsymbol{\alpha}_{k}\right) \boldsymbol{y}_{j}
$$

${ }^{a}$ e-mail: ian.smith@npl.co.uk 
be its location in the $k$ th position of the ball plate, $j=1, \ldots, n_{Y}, k=1, \ldots, n_{T}$. Here $T(\boldsymbol{y}, \boldsymbol{t})$ represents a rigid body transformation defined by six parameters $\boldsymbol{t}$, three translation parameters and three rotation angles. The measurement equations are of the form

$$
\boldsymbol{x}_{i}=\boldsymbol{y}_{j, k}+\boldsymbol{e}\left(\boldsymbol{x}_{i}, \boldsymbol{b}\right)+\boldsymbol{\epsilon}_{i}, \quad i \in I=\{1, \ldots, m\} .
$$

If the $3 \times 1$ random effects $\boldsymbol{\epsilon}_{i}$ are modelled as draws from a Gaussian distribution $\boldsymbol{\epsilon}_{i} \in \mathrm{N}\left(0, \sigma_{M}^{2} I\right)$, then estimates of the model parameters $\boldsymbol{y}_{j}, \boldsymbol{t}_{k}$, and $\boldsymbol{a}$ are determined by minimising

$$
\sum_{i=1}^{m} \boldsymbol{f}_{i}\left(\boldsymbol{y}_{j}, \boldsymbol{t}_{k}, \boldsymbol{b}\right)^{\mathrm{T}} \boldsymbol{f}_{i}\left(\boldsymbol{y}_{j}, \boldsymbol{t}_{k}, \boldsymbol{b}\right)
$$

where

$$
\boldsymbol{f}_{i}\left(\boldsymbol{y}_{j}, \boldsymbol{t}_{k}, \boldsymbol{b}\right)=\boldsymbol{x}_{i}-\boldsymbol{y}_{j, k}-\boldsymbol{e}\left(\boldsymbol{x}_{i}, \boldsymbol{b}\right),
$$

a nonlinear least squares problem. If $J$ is the Jacobian matrix of partial derivatives of $\boldsymbol{f}_{i}$ with respect to the optimisation parameters evaluated at the solution, the variance matrix $V$ for the fitted parameters is estimated by $V=\left(J^{\mathrm{T}} J\right)^{-1}$. The variance matrix $V_{\boldsymbol{b}}$ associated with the kinematic error parameters $\boldsymbol{b}$ is a submatrix of $V$.

In the following, we assume that estimates of the kinematic error parameters, $\boldsymbol{b}$, have been determined and that, following the implementation of an error correction, $\boldsymbol{b}$ is assigned a multivariate Gaussian distribution. We assume further that $\boldsymbol{e}(\boldsymbol{x}, \mathbf{0})=\mathbf{0}$. Similarly, we also assume that the random effects are also described by a Gaussian distribution:

$$
\boldsymbol{b} \sim \mathrm{N}\left(\mathbf{0}, V_{\boldsymbol{b}}\right), \quad \boldsymbol{\epsilon}_{i} \in \mathrm{N}\left(\mathbf{0}, \sigma_{M}^{2} I\right)
$$

Suppose measured data $\boldsymbol{x}_{i}, i \in I=1, \ldots, m$, is generated according to the model defined by

$$
\boldsymbol{x}_{i}=\boldsymbol{x}_{i}^{*}+\boldsymbol{e}\left(\boldsymbol{x}_{i}, \boldsymbol{b}\right)+\boldsymbol{\epsilon}_{i}
$$

where $\boldsymbol{b}$ and $\boldsymbol{\epsilon}_{i}$ are characterised as in expressions (2). Then, to first order, the $3 m \times 1$ vector of actual probe centres $\boldsymbol{x}_{I}^{*}$ is associated with the multivariate Gaussian distribution

$$
\boldsymbol{x}_{I}^{*} \mid \boldsymbol{x}_{I} \sim \mathrm{N}\left(\boldsymbol{x}_{I}, V_{I}\right),
$$

where the variance matrix $V_{I}$ is given by

$$
V_{I}=S_{\boldsymbol{b}} V_{\boldsymbol{b}} S_{\boldsymbol{b}}^{\mathrm{T}}+\sigma_{M}^{2} I
$$

and $S_{\boldsymbol{b}}$ is the $3 m \times p$ matrix of partial derivatives of $\boldsymbol{e}\left(\boldsymbol{x}_{i}, \boldsymbol{b}\right)$ with respect to the error parameters $\boldsymbol{b}$ evaluated at $\boldsymbol{b}=\mathbf{0}$. Equation (4) decomposes the variance associated with the best estimate $\boldsymbol{x}_{I}$ as the sum of the parametric error and random contributions. If $V_{\boldsymbol{b}}$ has Cholesky decomposition [8] $V_{\boldsymbol{b}}=L_{\boldsymbol{b}} L_{\boldsymbol{b}}^{\mathrm{T}}$, then

$$
V_{I}=G G^{\mathrm{T}}+\sigma_{M}^{2} I, \quad G=S_{\boldsymbol{b}^{L}} \boldsymbol{b}
$$

\section{Ordinary least squares (OLS) feature assessment}

Suppose $\boldsymbol{u} \mapsto \boldsymbol{s}(\boldsymbol{u}, \boldsymbol{a})$ defines a parametric curve or surface. The parameters $\boldsymbol{u}$ determine the position of a point on the surface and the parameters $\boldsymbol{a}$ determine the shape and position of the surface. We now assume that set of measured coordinates, $\boldsymbol{x}_{I}$ generated according to the model (3) is such that

$$
\boldsymbol{x}_{i}^{*}=\boldsymbol{s}\left(\boldsymbol{u}_{i}, \boldsymbol{a}\right)
$$

for some $\boldsymbol{u}_{i}$ and some $\boldsymbol{a}$.

The ordinary least squares (OLS) best-fit surface can be found by minimising

$$
\sum_{i=1}^{m} d^{2}\left(\boldsymbol{x}_{i}, \boldsymbol{a}\right), \quad d\left(\boldsymbol{x}_{i}, \boldsymbol{a}\right)=\left(\boldsymbol{x}_{i}-\boldsymbol{s}\left(\boldsymbol{u}_{i}^{*}, \boldsymbol{a}\right)\right)^{\mathrm{T}} \boldsymbol{n}_{i},
$$

where $\boldsymbol{u}_{i}^{*}$ specifies the point $\boldsymbol{s}_{i}^{*}=\boldsymbol{s}\left(\boldsymbol{u}_{i}^{*}, \boldsymbol{a}\right)$ on the surface closest to $\boldsymbol{x}_{i}$ and $\boldsymbol{n}_{i}$ is the normal vector at $\boldsymbol{s}_{i}^{*}$. For standard geometric elements, $d(\boldsymbol{x}, \boldsymbol{a})$ can be evaluated analytically. For more general surfaces, numerical methods are required [7]. Let $J$ be the Jacobian matrix associated with $d\left(\boldsymbol{x}_{i}, \boldsymbol{a}\right)$ at the solution $\boldsymbol{a}^{*}$ and let

$$
J=Q R=\left[\begin{array}{ll}
Q_{1} & Q_{2}
\end{array}\right]\left[\begin{array}{c}
R_{1} \\
\mathbf{0}
\end{array}\right]
$$

be its QR factorisation [8]. Then, to first order, $\boldsymbol{a}=$ $\boldsymbol{a}^{*}-R_{1}^{-1} Q_{1}^{\mathrm{T}} \boldsymbol{h}_{I}$, where

$$
\boldsymbol{h}_{i}=\boldsymbol{n}_{i}^{\mathrm{T}}\left(\boldsymbol{e}\left(\boldsymbol{x}_{i}, \boldsymbol{b}\right)+\boldsymbol{\epsilon}_{i}\right) .
$$

If $N$ is the $m \times 3 m$ block diagonal matrix storing $\boldsymbol{n}_{i}^{\mathrm{T}}$ in the $1 \times 3$ diagonal blocks, the sensitivity matrix $S_{\boldsymbol{a}}$ of the OLS fitted parameters $\boldsymbol{a}$ with respect to the random effects $\boldsymbol{e}_{I}$ is given by

$$
S_{\boldsymbol{a}}=-R_{1}^{-1} Q_{1}^{\mathrm{T}} N
$$

so that the variance matrix associated with the $\boldsymbol{x}_{I}$ is propagated through to the variance matrix associated with $\boldsymbol{a}$ according to

$$
V_{\boldsymbol{a}}=S_{\boldsymbol{a}} V_{I} S_{\boldsymbol{a}}^{\mathrm{T}}=S_{\boldsymbol{a}} S_{\boldsymbol{b}} V_{\boldsymbol{b}} S_{\boldsymbol{b}}^{\mathrm{T}} S_{\boldsymbol{a}}^{\mathrm{T}}+\sigma_{M}^{2}\left(R_{1}^{\mathrm{T}} R_{1}\right)^{-1}
$$

The second term on the right is the contribution of the random effects to the variance matrix associated with fitted parameters $\boldsymbol{a}$ and is the term that is usually evaluated in a nonlinear least squares model fit (see, e.g., $[5,4]$ ). The first term on the right is the contribution from the parametric errors. The matrix $S_{\boldsymbol{a}} S_{\boldsymbol{b}}$ is the sensitivity of the fitted parameters $a_{j}$ to the parametric errors $b_{k}$.

To first order the residual error vector is given by $\boldsymbol{d}=Q_{2} Q_{2}^{\mathrm{T}} \boldsymbol{h}_{I}$ with $\boldsymbol{h}_{I}$ as specified in expression (9) and the associated variance matrix is

$$
V_{\boldsymbol{d}}=S_{\boldsymbol{d}} V_{I} S_{\boldsymbol{d}}^{\mathrm{T}}, \quad S_{\boldsymbol{d}}=Q_{2} Q_{2}^{\mathrm{T}} N .
$$

As for $V_{\boldsymbol{a}}$, if $V_{I}$ is of the form (4), then $V_{\boldsymbol{d}}$ is similarly decomposed into contributions arising from parametric errors and random effects. 
Table 1. Sensitivities of cylinder parameters to parametric errors.

\begin{tabular}{|c|r|r|r|r|r|r|}
\hline$\partial a_{j} / \partial b_{k}$ & $b_{x x}$ & $b_{y y}$ & $b_{z z}$ & $b_{x y}$ & $b_{x z}$ & $b_{y z}$ \\
\hline$x_{0}$ & 60 & 0 & 0 & 60 & 0 & 0 \\
$y_{0}$ & 0 & 60 & 0 & 0 & 0 & 0 \\
$\alpha$ & 0 & 0 & 0 & 0 & 0 & 1 \\
$\beta$ & 0 & 0 & 0 & 0 & -1 & 0 \\
$r_{0}$ & 25 & 25 & 0 & 0 & 0 & 0 \\
\hline
\end{tabular}

Table 2. Sensitivities of residual distances to parametric errors.

\begin{tabular}{|c|r|r|r|r|r|r|}
\hline$\partial d_{i} / \partial b_{k}$ & $b_{x x}$ & $b_{y y}$ & $b_{z z}$ & $b_{x y}$ & $b_{x z}$ & $b_{y z}$ \\
\hline$d_{1}$ & 25 & -25 & 0 & 0 & 0 & 0 \\
$d_{2}$ & -20 & 20 & 0 & 15 & 0 & 0 \\
$d_{3}$ & 8 & -8 & 0 & -24 & 0 & 0 \\
$d_{4}$ & 8 & -8 & 0 & 24 & 0 & 0 \\
$d_{5}$ & -20 & 20 & 0 & -15 & 0 & 0 \\
\hline
\end{tabular}

\subsection{Example: effect of scale and squareness on cylindricity assessment}

To illustrate this approach, we consider a model of a CMM with scale and squareness errors $[2,3]$ so that

$$
\boldsymbol{e}(\boldsymbol{x}, \boldsymbol{b})=\left[\begin{array}{ccc}
b_{x x} & b_{x y} & b_{x z} \\
0 & b_{y y} & b_{y z} \\
0 & 0 & b_{z z}
\end{array}\right] \boldsymbol{x}
$$

The diagonal elements represent the scale errors along the three axis while the non-zero off-diagonal elements are the squareness errors. We assume there are 15 measured points on a cylinder of radius $50 \mathrm{~mm}$, five points on three circles $25 \mathrm{~mm}$ apart. We assume that $\sigma_{M}=0.0005 \mathrm{~mm}$ and that

$$
\boldsymbol{b}=\left(b_{x x}, b_{y y}, b_{z z}, b_{x y}, b_{x z}, b_{y z}\right)^{\mathrm{T}}
$$

is associated with $\mathrm{N}\left(\mathbf{0}, \sigma^{2} I\right)$ with $\sigma=2 \times 10^{-5}$. For this statistical model, the standard uncertainties associated with the point coordinates are of the order of $0.002 \mathrm{~mm}$.

A cylinder can be specified by five parameters $\boldsymbol{a}=$ $\left(x_{0}, y_{0}, \alpha, \beta, r_{0}\right)^{\mathrm{T}}$, where $x_{0}$ and $y_{0}$ are the coordinates of the point of intersection with the plane $z=0$, the rotation angles $\alpha$ and $\beta$ determine the axis direction and $r_{0}$ is the radius. For this calculation, $\boldsymbol{a}=$ $(60,60,0,0,25)^{\mathrm{T}}$. Table 1 gives the sensitivities of the fitted parameters with respect to the parametric errors. The scale errors have an effect on the location and radius parameters; the $x y$-squareness parameters $b_{x y}$ has an effect on the $y$-coordinate of the axis location while the squareness errors $b_{x z}$ and $b_{y z}$ have an effect on the axis direction. Table 2 gives the sensitivities of the residual distances associated with the five points on the middle circle; sensitivities for points on the other two circles are the same. The table shows that only the $x$ - and $y$-scale errors along with the $x y$ squareness have an effect on the evaluated form error.

\section{Generalised least squares (GLS) feature assessment}

The OLS feature assessment corresponds to the maximum likelihood estimate of the feature parameters $\boldsymbol{a}$ for the case $V_{I}=\sigma_{M} I$. For more general variance matrices, the maximum likelihood estimate is given by the solution of

$$
\min _{\boldsymbol{a},\left\{\boldsymbol{u}_{i}\right\}}\left(\boldsymbol{x}_{I}-\boldsymbol{s}_{I}\right) V_{I}^{-1}\left(\boldsymbol{x}_{I}-\boldsymbol{s}_{I}\right),
$$

where

$$
s_{I}=\left[\begin{array}{c}
s\left(u_{1}, a\right) \\
s\left(u_{2}, a\right) \\
\vdots \\
s\left(u_{m}, a\right)
\end{array}\right]
$$

Even for standard geometric features, there is in general no analytical expression for the optimal $\boldsymbol{u}_{i}^{*}$. However, if $V_{I}$ has the form as in expression (5),

$$
V_{I}=G G^{\mathrm{T}}+\sigma_{M}^{2} I, \quad G=\left[\begin{array}{c}
G_{1} \\
G_{2} \\
\vdots \\
G_{M}
\end{array}\right],
$$

where $G$ is a $3 m \times p$ matrix made up of $3 \times p$ blocks $G_{i}$, then the optimal $\boldsymbol{a}$ that solves problem (12) is also the optimal $\boldsymbol{a}$ for the problem

$$
\min _{\boldsymbol{a}, \boldsymbol{b},}\left\{\frac{1}{\sigma_{M}^{2}} \sum_{i \in I} d^{2}\left(\boldsymbol{x}_{i}-G_{i} \boldsymbol{b}, \boldsymbol{a}\right)\right\}+\boldsymbol{b}^{\mathrm{T}} \boldsymbol{b} .
$$

Thus, the optimal $\boldsymbol{u}_{i}$ defines the distance from the point $\boldsymbol{x}_{i}-G_{i} \boldsymbol{b}$ to the surface $\boldsymbol{u} \mapsto \boldsymbol{s}(\boldsymbol{u}, \boldsymbol{a})$. For standard geometric elements, this distance can be evaluated analytically. Problem (13) is a standard nonlinear least squares problem that can be solved using the Gauss-Newton algorithm in which it is required to evaluate

$$
\begin{aligned}
J_{i j} & =\frac{\partial d}{\partial a_{j}}\left(\boldsymbol{x}_{i}-G_{i} \boldsymbol{b}, \boldsymbol{a}\right), \\
K_{i k} & =\frac{\partial d}{\partial b_{k}}\left(\boldsymbol{x}_{i}-G_{i} \boldsymbol{b}, \boldsymbol{a}\right)=-\boldsymbol{n}_{i}^{\mathrm{T}} G_{i},
\end{aligned}
$$

where $\boldsymbol{n}_{i}$ is the $3 \times 1$ vector of partial derivatives of $d(\boldsymbol{x}, \boldsymbol{a})$ evaluated at $\boldsymbol{x}=\boldsymbol{x}_{i}-G_{i} \boldsymbol{b}$. Compared to the OLS fit, the only new information required is the evaluation of $\boldsymbol{n}_{i}$, a straightforward calculation. If required, the complex-step method can be used to provide an accurate numerical estimate of these derivatives [1, 10]. For example, if the software that evaluates $d(\boldsymbol{x}, \boldsymbol{a})$ supports complex arithmetic, then the imaginary part of $d(\boldsymbol{x}+i h \boldsymbol{e}, \boldsymbol{a}) / h$, where $\boldsymbol{e}=(1,0,0)^{\mathrm{T}}, i=\sqrt{-1}$, and $h=10^{-100}$, evaluates $\partial d / \partial x$, etc.

The variance matrix $\tilde{V}$ associated with the fitted parameters according to problem (13) is given by

$$
\tilde{V}=\left(\tilde{J}^{\mathrm{T}} \tilde{J}\right)^{-1}, \quad \tilde{J}=\left[\begin{array}{cc}
J / \sigma_{M} & K / \sigma_{M} \\
\mathbf{0} & I
\end{array}\right],
$$

where $J$ and $K$ are defined as in expressions (14), evaluated at the solution. 
Table 3. Uncertainties associated with fitted parameters.

\begin{tabular}{|c|r|r|r|}
\hline $\boldsymbol{a}$ & $\boldsymbol{a}_{\mathrm{OLS}}$ & $\boldsymbol{a}_{\mathrm{GLS}}$ & $\boldsymbol{a}_{\mathrm{GLS}, \mathrm{C}}$ \\
\hline$x_{0} / \mu \mathrm{m}$ & 1.7 & 1.0 & 0.6 \\
$y_{0} / \mu \mathrm{m}$ & 1.3 & 0.9 & 0.5 \\
$\alpha / \operatorname{arcsecond}$ & 4.5 & 4.5 & 4.5 \\
$\beta /$ arcsecond & 4.5 & 4.5 & 4.5 \\
$r_{0} / \mu \mathrm{m}$ & 0.7 & 0.7 & 0.3 \\
\hline
\end{tabular}

The advantage of the GLS formulation (12) is that the uncertainty information is used more effectively so that the uncertainties associated with the fitted parameters are smaller that those associated with the OLS fitted parameters as evaluated in expression (10). In terms of $J$ and $K$, we have

$$
V \boldsymbol{a}_{\text {OLS }}=\sigma_{M}^{2}\left(J^{\mathrm{T}} J\right)^{-1}+\left(J^{T} J\right)^{-1} J^{\mathrm{T}} K^{\mathrm{T}} K J\left(J^{\mathrm{T}} J\right)^{-1},
$$

while

$V \boldsymbol{a}_{\mathrm{GLS}}=\sigma_{M}^{2}\left(J^{\mathrm{T}} J\right)^{-1}+\left(J^{\mathrm{T}} J\right)^{-1} J^{\mathrm{T}} K^{\mathrm{T}} M^{-1} K J\left(J^{\mathrm{T}} J\right)^{-1}$, with

$$
M=I+\frac{1}{\sigma_{M}^{2}} K^{\mathrm{T}}\left[I-J\left(J^{\mathrm{T}} J\right)^{-1} J^{\mathrm{T}}\right] K .
$$

Thus $V \boldsymbol{a}_{\mathrm{OLS}}$ is the same as $V \boldsymbol{a}_{\mathrm{GLS}}$ but with $M$ replaced by $I$. Since $M-I$ is positive semi-definite, $V_{\boldsymbol{a}_{\mathrm{OLS}}}-V_{\boldsymbol{a}_{\mathrm{GLS}}}$ must also be positive semi-definite.

Table 3 shows the uncertainties associated with the parameters of a cylinder fitted to data generated according to the model described in section 3.1 using OLS and GLS fits. The uncertainties associated with $x_{0}$ and $y_{0}$ are significantly smaller for the GLS fit. The eigenvalues of the matrix $M^{-1}$ are $0.06,0.12,1.0,1.0$, 1.0 , and 1.0, showing that in a two dimensional subspace, the uncertainty contribution from the systematic scale and squareness effects is much reduced when the GLS fit is used.

\subsection{Comparator measurements}

The advantage of formulation (13), compared to formulation (12), is that the systematic effects $\boldsymbol{b}$ appear explicitly allowing updated estimates of the parameters to be obtained. This advantage opens the way to evaluating the reduction in uncertainties that can be achieved when a CMM is used in comparator mode.

For example, suppose a CMM with scale and squareness errors as described in section 3.1 is used to measure a calibrated ring gauge. We assume that the uncertainty associated with the calibration value of the gauge radius is $0.0002 \mathrm{~mm}$ and the form errors of the gauge are drawn from a Gaussian distribution with standard deviation $\sigma_{F}=0.0002 \mathrm{~mm}$ [6]. This calibration information allows us to derive improved estimates of the $x$ - and $y$-axis scale errors and the $x y$-squareness parameters. The uncertainties associated with these estimates are reduced from $2 \times 10^{-5}$ to $6.0 \times 10^{-6}$ for the $x$ - and $y$-axis scale errors and $7.3 \times 10^{-6}$ for the $x y$-squareness error. The fourth column in table 4 shows the uncertainties in the fitted parameters for the measurement of a ring gauge using these improved estimates.

\section{Summary and concluding remarks}

This paper has been concerned with fitting geometric elements to coordinate data that has an associated uncertainty structure that reflects the systematic effects associated with the measuring system. The uncertainty structure allows us to determine the generalised least squares estimate in a way that is computationally efficient and requires only a small modification to software that implements a standard least squares fit. The approach also allow determines fitted surfaces with associated uncertainties for a CMM used in comparator mode.

\section{Acknowledgements}

This work was supported by the UK's National Measurement Systems programmes for Engineering Measurement and Mathematics and Modelling.

\section{References}

1. R. Boudjemaa, M. G. Cox, A. B. Forbes, and P. M. Harris. Automatic differentiation and its applications to metrology. In P. Ciarlini, M. G. Cox, F. Pavese, and G. B. Rossi, editors, Advanced Mathematical and Computational Tools in Metrology VI, pages 170-179, Singapore, 2004. World Scientific.

2. A. B. Forbes. Surface fitting taking into account uncertainty structure in coordinate data. Measurement Science and Technology, 17:553-558, 2006.

3. A. B. Forbes. Uncertainty evaluation associated with fitting geometric surfaces to coordinate data. Metrologia, 43(4):S282-S290, August 2006.

4. A. B. Forbes. Nonlinear least squares and Bayesian inference. In F. Pavese, M. Bär, A. B. Forbes, J.-M. Linares, C. Perruchet, and N.-F. Zhang, editors, Advanced Mathematical and Computational Tools in Metrology VIII, pages 103111, Singapore, 2009. World Scientific.

5. A. B. Forbes. Parameter estimation based on least squares methods. In F. Pavese and A. B. Forbes, editors, Data modeling for metrology and testing in measurement science, pages 147-176, New York, 2009. Birkhäuser-Boston.

6. A. B. Forbes. Uncertainty associated with form assessment in coordinate metrology. Int. J. of Metrol. and Qual. Eng., 4:17-22, 2013.

7. A. B. Forbes and H. D. Minh. Form assessment in coordinate metrology. In E. H. Georgoulis, A. Iske, and J. Levesley, editors, Approximation Algorithms for Complex Systems, Springer Proceedings in Mathematics, Vol 3, pages 69-90, Heidelberg, 2011. Springer-Verlag.

8. G. H. Golub and C. F. Van Loan. Matrix Computations. John Hopkins University Press, Baltimore, third edition, 1996.

9. H. Kunzmann, E. Trapet, and F. Waldele. A uniform concept for calibration, acceptance test 


\section{$16^{\text {th }}$ International Congress of Metrology}

and periodic inspection of co-ordinate measuring machines using reference objects. Annals of the CIRP, 39:561-564, 1990.

10. W. Squire and G. Trapp. Using complex variables to estimate derivatives of real functions. SIAM Rev., 40:110-112, 1998.

11. G. Zhang, R. Ouyang, B. Lu, R. Hocken, R. Veale, and A. Donmez. A displacement method for machine geometry calibration. Annals of the CIRP, 37:515-518, 1988. 